\title{
Latent Fingerprint Training with NIST Special Database 27 and Universal Latent Workstation
} NISTIR 6799

\author{
Michael D. Garris
}

National Institute of Standards and Technology

Building 225, Room A216

Gaithersburg, MD 20899 



\title{
Latent Fingerprint Training with NIST Special Database 27 and Universal Latent Workstation
}

\author{
Michael D. Garris (mgarris@nist.gov)
}

\begin{abstract}
The National Institute of Standards and Technology, in collaboration with the FBI, has published NIST Special Database 27 (SD27) "Fingerprint Minutiae from Latent and Matching Tenprint Images." This CD-ROM collection contains images of 258 latent crime scene fingerprints and their matching rolled tenprints. In addition, minutiae features validated by a team of professional latent examiners are provided for each fingerprint. Meanwhile, the FBI has also developed the Universal Latent Workstation (ULW). This workstation has been designed to render and enhance fingerprint images, assist the operator in labeling minutiae and other fingerprint features, and formatting this information into a standard transaction file for searching federal, state, and local law enforcement fingerprint repositories. Using the ULW in conjunction with SD27 poses a powerful and inexpensive training tool for fingerprint examiners. This report documents the steps needed to load SD27 fingerprint images into ULW, and how trainee results can then be overlaid with the validated minutiae in SD27. Given these steps, a variety of training scenarios are possible.
\end{abstract}

Keywords: ANSI/NIST, database, FBI, fingerprint, grayscale, IAFIS, image, latent, minutiae, tenprint, training, ULW

\section{INTRODUCTION}

The National Institute of Standards and Technology (NIST), in collaboration with the Federal Bureau of Investigation (FBI), has published a database of grayscale fingerprint images and corresponding minutiae data. The database, NIST Special Database 27 (SD27) "Fingerprint Minutiae from Latent and Matching Tenprint Images," contains latent fingerprints from crime scenes and their matching rolled fingerprint mates. [1] In all there are 258 latent cases. Each case includes the latent image, the matching tenprint image, and four sets of minutiae that have been validated by a professional team of latent examiners.

Meanwhile, the Criminal Justice Information Services (CJIS) division of the FBI has developed an interactive software tool called the Universal Latent Workstation (ULW). This program, developed to run on the Microsoft family of Win32 computers ${ }^{1}$, allows fingerprint examiners to encode fingerprint features so that they can be searched against federal, state, or local fingerprint repositories. The software displays the fingerprint image, providing image processing tools to improve the quality of the rendered fingerprint. It also incorporates feature extraction tools that can be used to assist fingerprint examiners in labeling features prior to search.

\footnotetext{
${ }^{1}$ Specific software products and equipment identified in this paper were used in order to adequately support the development of the application described in this document. In no case does such identification imply recommendation or endorsement by the National Institute of Standards and Technology, nor does it imply that the software or equipment identified is necessarily the best available for the purpose.
} 

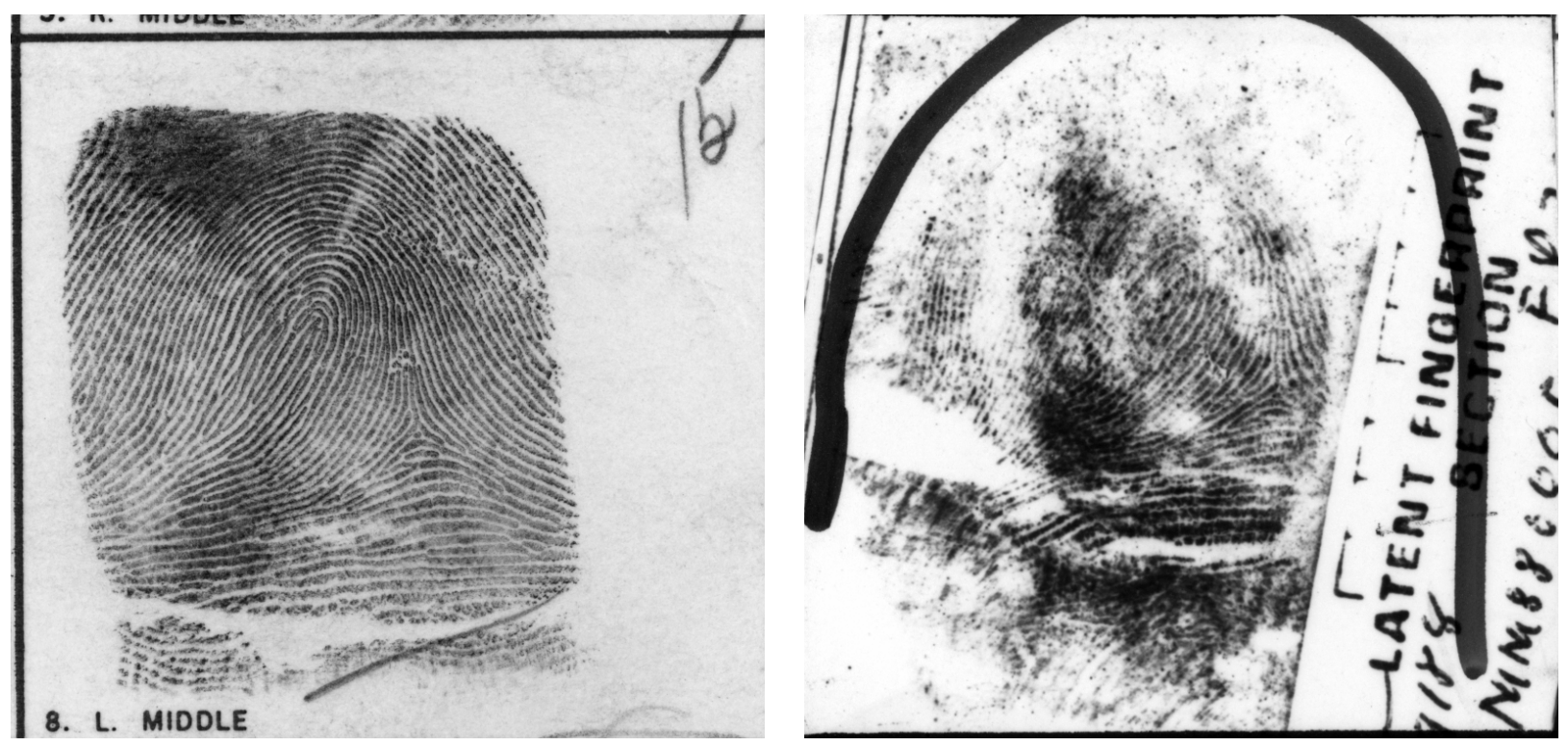

Figure 1. Tenprint (left) with matching latent (right).

Together, these two resources represent an effective tool for training latent fingerprint examiners. The following training scenario is possible. A fingerprint image from SD27 can be displayed by ULW. A trainee then uses ULW to label the features, including minutiae, on the fingerprint. The SD27 feature data for the particular fingerprint (validated by professional latent examiners) is then overlaid and compared with the trainees results. Similarly, results between a latent fingerprint and its matching tenprint can be compared and studied. This report documents how these activities are performed using SD27 in conjunction with ULW.

The left image in Figure 1 shows a typical fingerprint produced by inking and rolling the fingertip onto a fingerprint card. These types of fingerprints represent the bulk of the data submitted, stored, and searched at the FBI. They are commonly referred to as tenprints, as all ten fingers are usually captured. The right image in the figure shows a "good" quality latent that was produced by the same finger that created the tenprint on the left. As one would expect, the composition and quality of latent fingerprints are significantly different from tenprints. Typically, only a portion of the finger is present in the latent, the surface on which the latent was imprinted is unpredictable, and the clarity of friction skin details are often blurred or occluded. All this leads to fingerprints of significantly lesser quality than typical tenprints

Traditionally, two fingerprints have been compared using discrete features called minutiae. These features include points in a finger's friction skin where a ridge ends (called a ridge ending) or splits (called a ridge bifurcation). Typically, there are on the order of 100 to 200 minutiae on a tenprint. In order to search and match fingerprints, the coordinate location and the orientation of the ridge at each minutia point are recorded. Figure 2 shows an example of the two types of minutiae. The minutiae are marked in the right image, and the tails on the markers point in the direction of the minutia's orientation. 

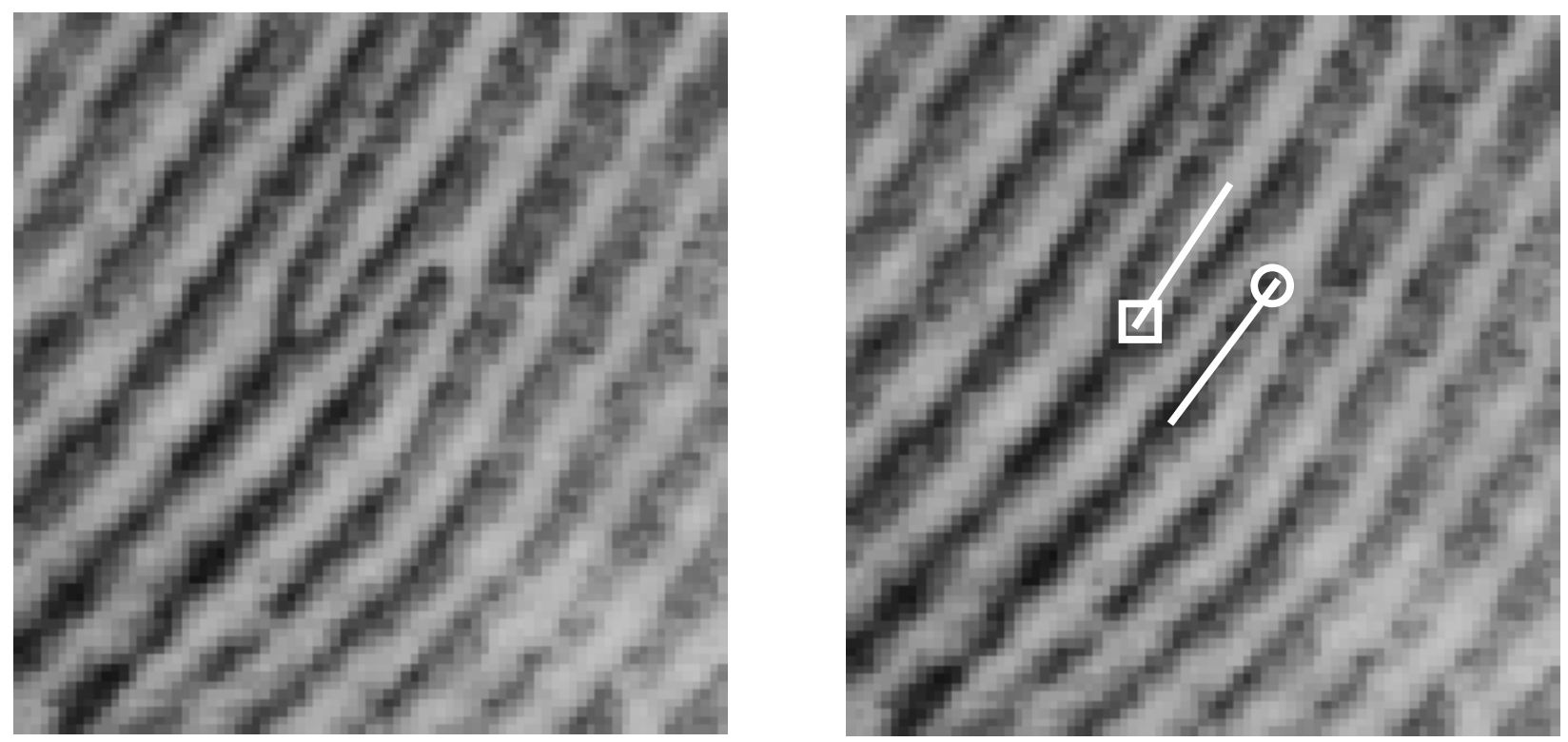

Figure 2. Minutiae: bifurcation (square marker) and ridge ending (circle marker)

Automated Fingerprint Identification Systems (AFIS) have been the subject of much research over the years [2]-[9], and today these systems are able to automatically and reliably capture, store, and search tenprints. Unfortunately, the image quality of most crime scene fingerprints is so poor that AFIS technology cannot be reliably applied to latent fingerprints. Yet there is an obvious need for law enforcement agencies to search tenprint repositories using fingerprints gathered at crime scenes. Although current technology may not automatically and reliably process latent fingerprints, computer technology can be used to assist a fingerprint examiner in his work. This is one of the primary goals behind ULW.

In order to successfully search a tenprint repository using a latent fingerprint, all the minutiae on the latent must be accurately labeled (encoded). Tenprints typically have more than 100 minutiae on them, so automatic AFIS systems have a generous number of features on which to work, and they are somewhat tolerant to a few missed or falsely labeled minutiae. Latents on the other hand are often only a portion of the fingertip, and they frequently contain as few as a dozen clear minutiae. Therefore, labeling every identifiable minutia is critical, and fingerprint examiners must go through significant training to develop expertise.

As was mentioned earlier, SD27 contains two sets of images (latents and tenprint mates) and four sets of minutiae validated by professional latent examiners. Of these four, each image has two sets of corresponding minutiae. The first is an ideal set, containing all the visible minutiae on the respective image. The second is a matched set, containing only those minutiae that are in common between the latent and tenprint images. For training purposes, the ideal set is probably of greater use. (Please note that cores and deltas are also included in the feature sets, and that only the ideal set of minutiae for latents makes a distinction between ridge endings and bifurcations.)

In addition to the training of fingerprint examiners, SD27 was also published for other purposes. One of these is the promotion and exhibition of new record types defined in the ANSI/NIST-ITL 1-2000 "Data Format for the Interchange of Fingerprint, Facial, Scar Mark \& Tattoo (SMT) Information" standard, commonly referred to as ANSI/NIST 2000. [10] This standard started out 
in 1986 primarily as a common interchange standard for transmitting minutiae data in an ASCII format. [11] In 1993, image records were adopted and became widely used. [12] These image records were binary formatted, and of these, the Type- 4 fingerprint record has been the most commonly used. In 2000, a set of tagged field image records was adopted to eventually replace the binary formatted records. Record Type-13 was specified to hold latent fingerprint images and record Type-14 was specified to hold tenprint fingerprint images. Changes were also made within the format of the Type-9 minutiae record. The ANSI/NIST data files in SD27 have been formatted using these new record types and formats in order to promote their use in future applications.

ULW is currently written to the FBI's Electronic Fingerprint Transmission Specification (Version 7) [13], which is based on the 1993 version of the ANSI/NIST standard and does not yet support the use of the new image records (Type-13 \& 14) in FBI fingerprint transaction processing. This was still the case as of July 2001 with the release of ULW (Version 2.9). As a result, the files distributed with SD27 cannot be opened directly as FBI fingerprint transactions in ULW, but must be first manipulated.

The remainder of this report provides instructions on the steps that must be taken to accomplish this. Section 2 describes the steps necessary to load an SD27 fingerprint image into a new fingerprint transaction in ULW. Section 3 describes how SD27 minutiae data can be compared against trainee results in ULW. These steps have been tested and proven to work on ULW (Versions 2.75 and 2.9). At a minimum, these steps require an installed copy of ULW, and a basic familiarity with the Microsoft ClipBook Viewer.

NIST Special Database 27 "Minutiae from Latents and Matching Tenprint Images" is distributed on CD-ROM by the Standard Reference Data Group at NIST. To get pricing information and/or to order the database, please look on the Web at www.nist.gov/srd/nistsd27.htm or contact:

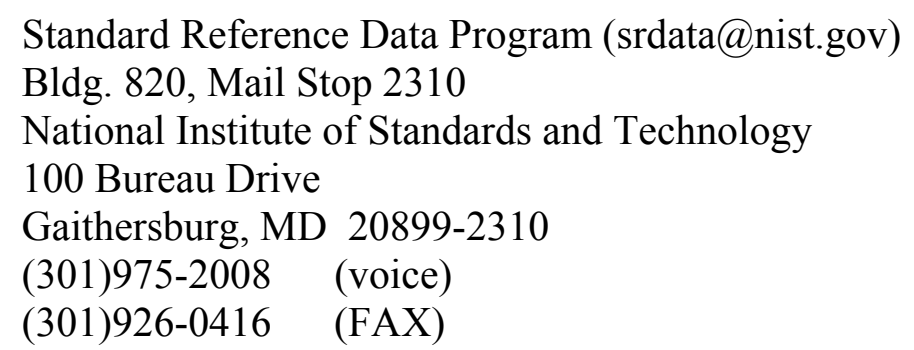

ULW is available free of charge through the FBI. To receive more information regarding ULW, please contact:

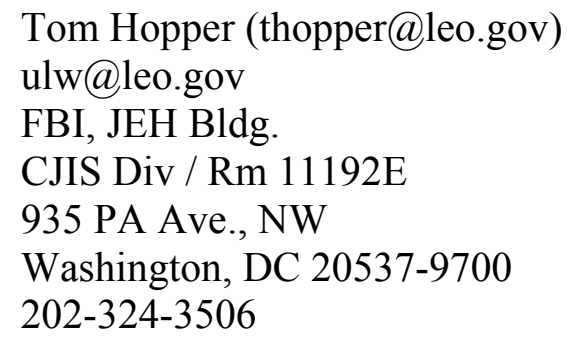




\section{LOADING SD27 FINGERPRINTS INTO ULW}

In order to open a ULW fingerprint transaction containing an SD27 fingerprint, the fingerprint image must first be placed on the Microsoft Clipboard; a new transaction in ULW is opened; and the image on the clipboard is pasted into ULW. This section describes this process, but in order to begin, an understanding of the organization of the data files distributed in SD27 is needed.

\subsection{SD27 Organization}

The ANSI/NIST data files on the SD27 CD-ROM are located in the top-level distribution directory Data. Under Data are three folders (Good, Bad, and Ugly) containing 88, 85, and 85 latent cases respectively. Based on the quality of the latent image, there is a folder in one of the quality directories for each latent case in the database. The names of these folders include a unique integer case identifier ranging from 1 to 300 .

Within each latent case there are six ANSI/NIST 2000 formatted files. The latent and tenprint mate images are in two separate files with extension "eft". The latent image is encapsulated in a tagged image Type- 13 record, and the tenprint mate is encapsulated in a tagged image Type-14 record. Each image has been scanned at 19.69 pixels per millimeter (500 pixels per inch), quantized to 256 levels of gray, and stored in an uncompressed format.

In addition to the two image files, there are four minutiae files corresponding to the ideal minutiae on the latent, the ideal minutiae on the tenprint mate, the matched minutiae on the latent, and the matched minutiae on the mate. All four files have the extension "lff". Each set of minutiae is stored in a tagged field Type-9 record. Figure 3 illustrates the data directory hierarchy.

SD27 uses a fixed file naming convention. An example of this convention can be seen in Figure 3 with the six filenames listed under the latent case directory G001. The naming convention is documented in Figure 4. 


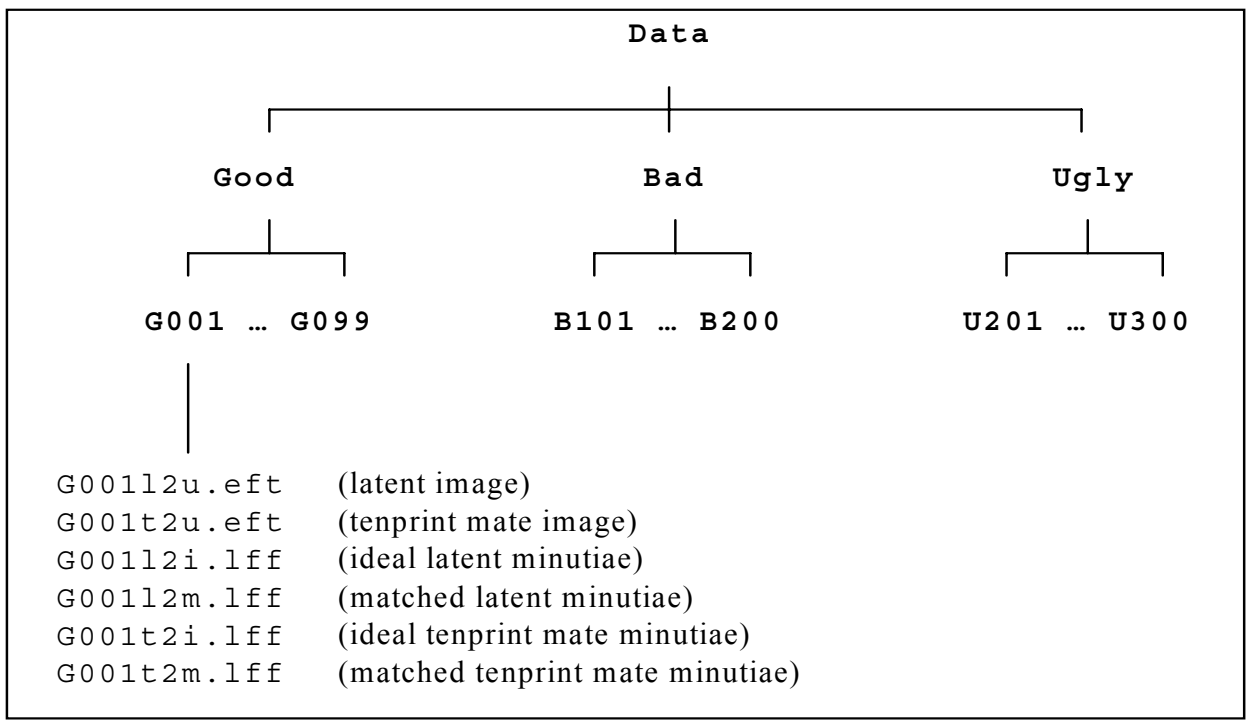

Figure 3. SD27 Data directory

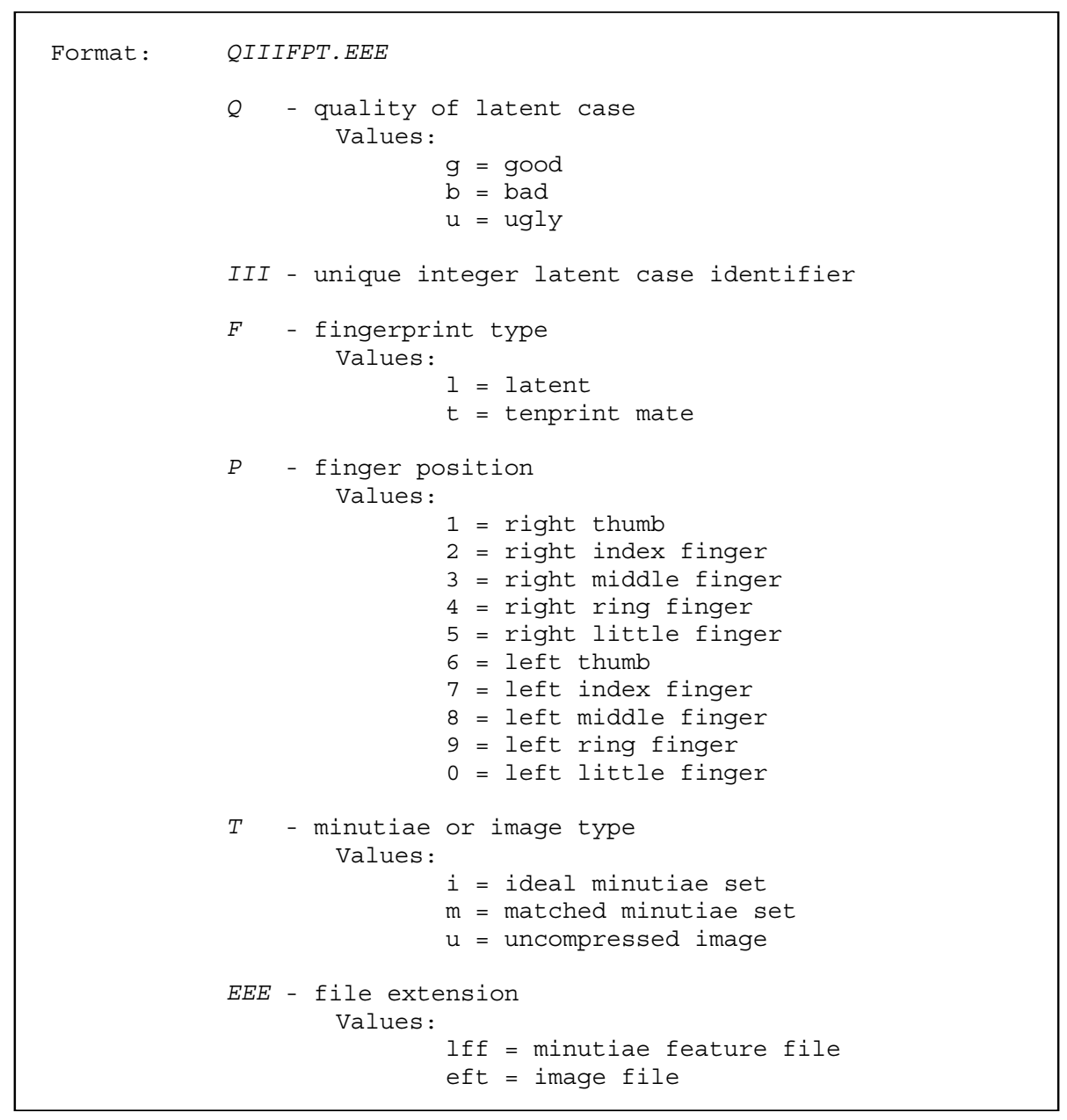

Figure 4. SD27 naming convention 


\subsection{Open SD27 Fingerprint Image}

All ANSI/NIST files in SD27 with filename extension "eft" contain fingerprint image records and can be displayed using the following steps in ULW. To start, use the "Oppen EFTS File" option in ULW under the main File menu.

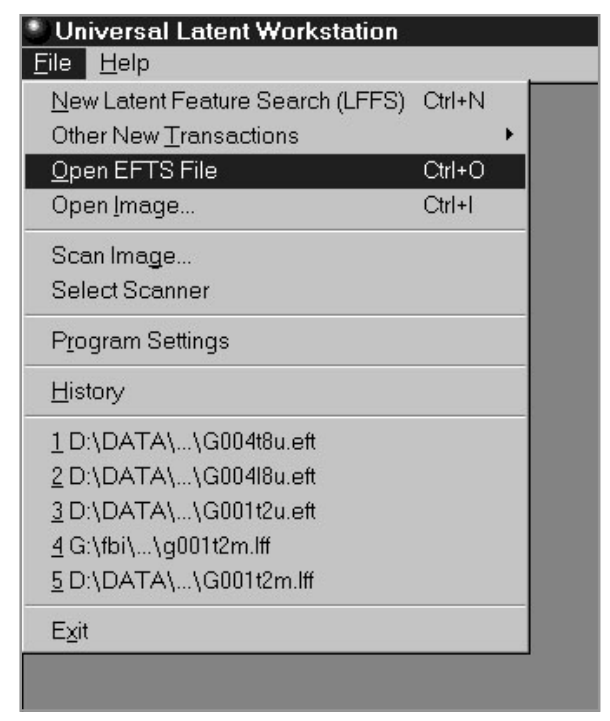

Figure 5. Oppen operation

A Windows Explorer dialogue box will pop up. To select a data file to open, use the Look in: pull-down to change drives to the CD-ROM drive on which SD27 is mounted. From there, select the folder Data, then a subfolder from the list Good, Bad, or Ugly, and then a case folder. In the example below, the case folder G004 is selected.

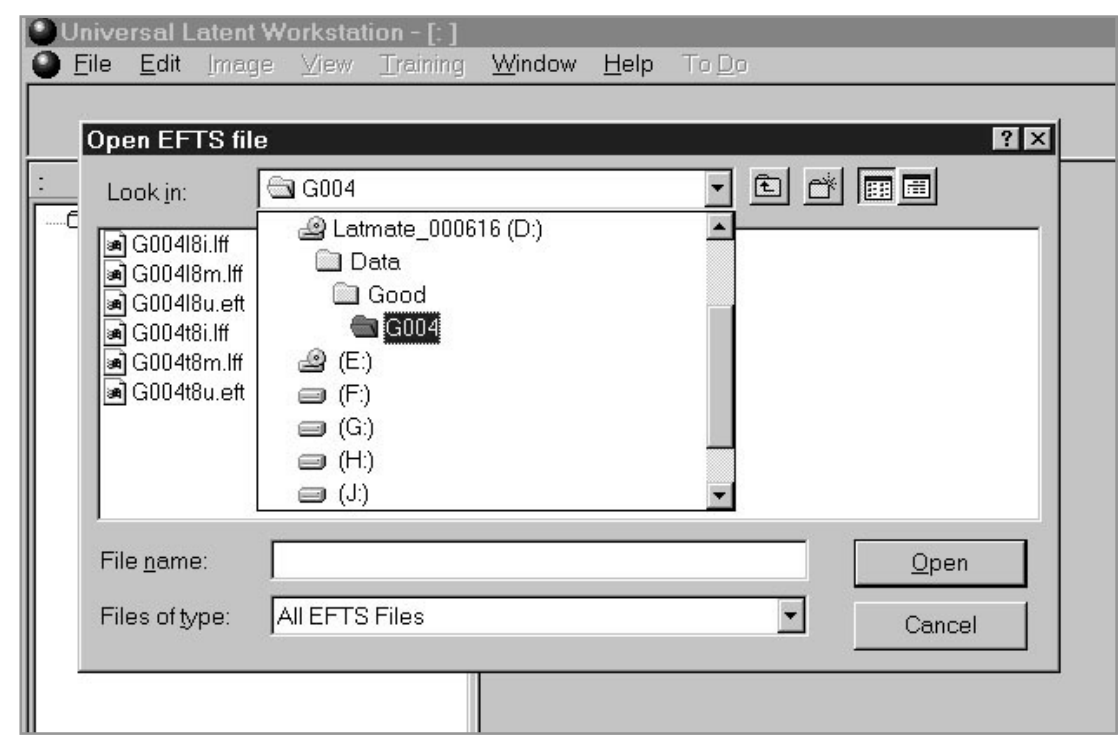

Figure 6. Select an SD27 case folder G004 
At this point, either the latent or tenprint image file within case G004 may be selected. In this example, the ANSI/NIST file G00418u . eft containing the latent fingerprint image is chosen.

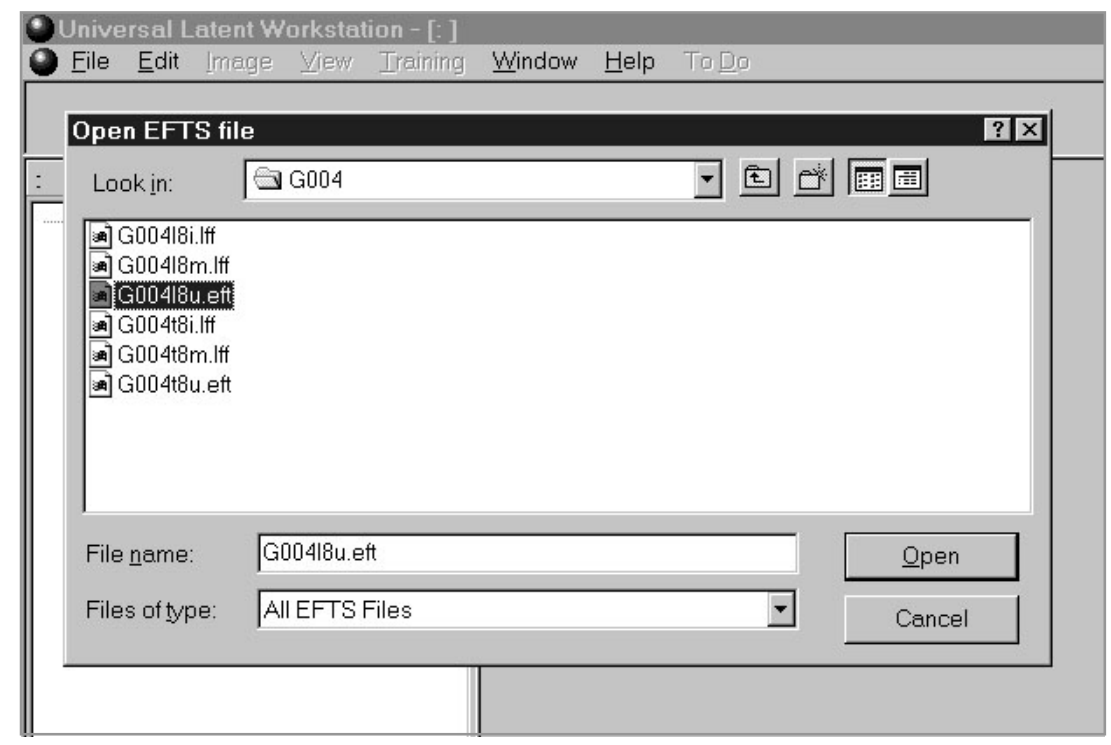

Figure 7. Select an SD27 latent fingerprint image file

The ANSI/NIST file is loaded into ULW, and the content of the text fields of the Type-13 record are listed to the right as shown below.

\begin{tabular}{|c|c|c|c|c|c|}
\hline \multicolumn{6}{|c|}{ O File Endit Image View Iraining Window Help ToDo } \\
\hline NISTDATA: G004I8u.eft & Name & Abbr & Field & $\#$ & Value \\
\hline \multirow{13}{*}{$\begin{array}{l}\text { 日 Rec 1: Transaction Information } \\
\text { C Transaction Information } \\
\text { C Rec 13: Latent Image } \\
\Rightarrow \text { Latent Image } \\
\text { O. Image }\end{array}$} & Logical Record Length & LEN & 13.001 & 1 & 614558 \\
\hline & Image Designation Character & IDC & 13.002 & 1 & 00 \\
\hline & Impression Type & IMP & 13.003 & 1 & 6 \{Latent photo $\}$ \\
\hline & Source Agency/ORl & SRC & 13.004 & 1 & DCFBIAFIS \\
\hline & Latent Capture Date & LCD & 13.005 & 1 & Jul 07, 1999 \\
\hline & Horizontal Line Length & HLL & 13.006 & 1 & 800 \\
\hline & Vertical Line Length & VLL & 13.007 & 1 & 768 \\
\hline & Scale Units & SLC & 13.008 & 1 & 2 \\
\hline & Horizontal Pixel Scale & HPS & 13.009 & 1 & 197 \\
\hline & Vertical Pixel Scale & VPS & 13.010 & 1 & 197 \\
\hline & Compression Algorithm & CGA & 13.011 & 1 & NONE \\
\hline & Bits Per Pixel & $\mathrm{BPX}$ & 13.012 & 1 & 8 \\
\hline & Finger Position & FGP & 13.013 & 1 & 08 \{Left middle\} \\
\hline
\end{tabular}

Figure 8. Listing of text fields for latent fingerprint Type-13 record 
To view the actual image, click on the Image field in the NISTDATA list as shown in Figure 8. ULW will then pop up a new window displaying the actual image as shown below.

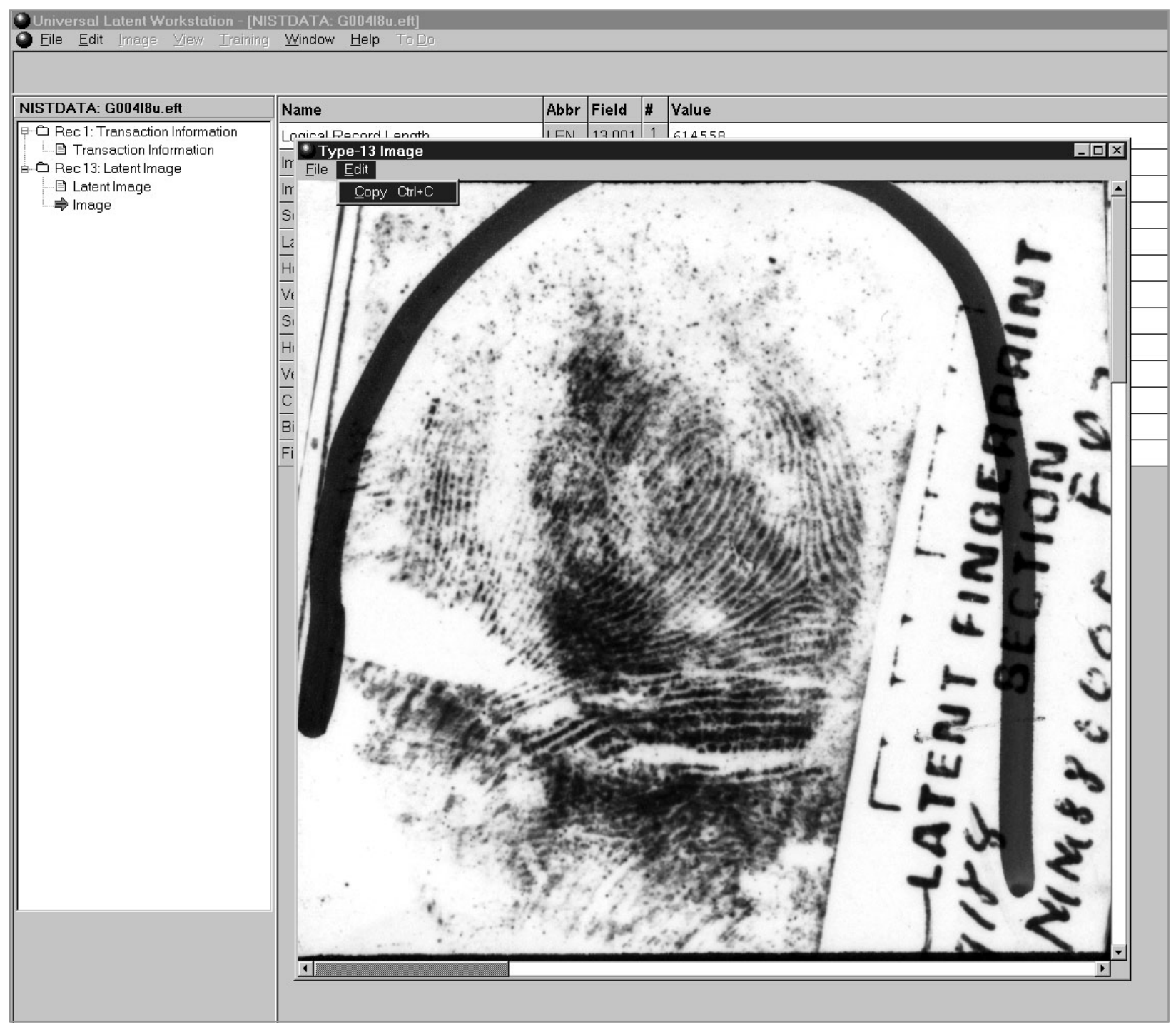

Figure 9. Separate image window displayed by ULW

\subsection{Copy Image from ULW to Clipboard}

Now that the image is displayed, it can be copied from ULW to the Microsoft Clipboard. It is highly recommended that the ClipBook Viewer be used in this process. To launch the viewer, click on the Start button on your Windows Task Bar and select the Run option. In the Run dialogue box that pops up, type in the program name clipbrd and then click OK. ${ }^{2}$

2 Note the computer system's display settings may affect the quality of the image stored in the clipboard. For example, if the display is set to High Color (16-bit), grayscale images that are stored in the clipboard are reduced in depth so that the image only contains 64 unique shades of gray instead of 256. Check the display settings under the system's Control Panel to ensure the display is set to True Color ( 32 bit), in which case images that are stored in the clipboard are not affected. 
It is highly recommended that you delete the current contents of the Clipboard (if any exists) before attempting to copy an image from ULW. To delete the Clipboard's contents, either click the Delete icon on the viewer's tool bar, or select the Delete option under the main Édit menu. Once the Clipboard is verified to be empty, click on the image window in ULW and then select the Copy option from the image window's Edit menu as shown in Figure 9.

The results of the copy can be verified using the ClipBook Viewer to make sure the image is on the clipboard as shown below.

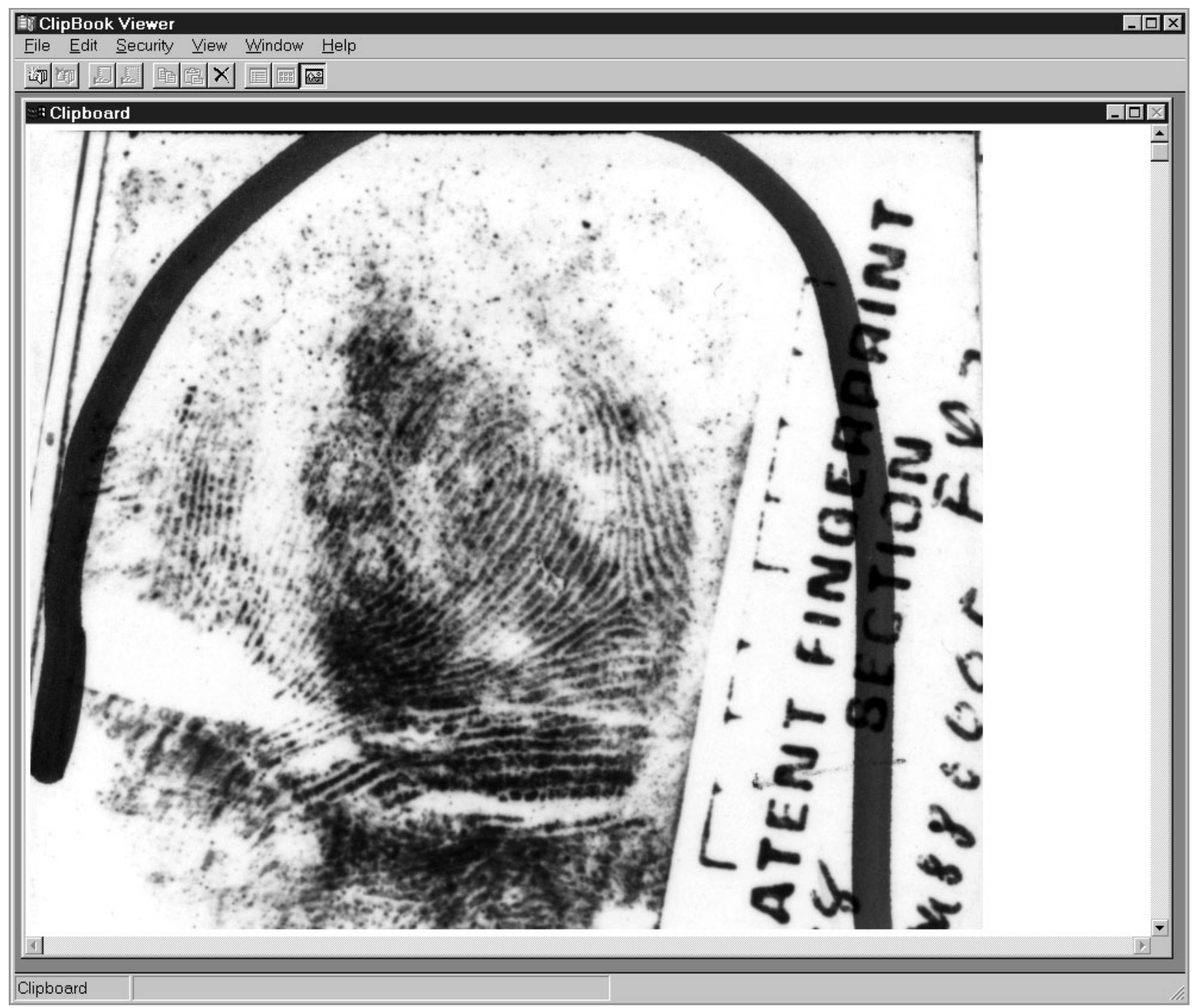

Figure 10. Image copied to Microsoft Clipboard

\subsection{Open New LFFS Transaction in ULW}

All the steps to this point are needed to copy a fingerprint image of interest onto the Microsoft Clipboard. With the image on the Clipboard, the current session in ULW can be closed by selecting the Close option under the main File menu of the image window displaying the

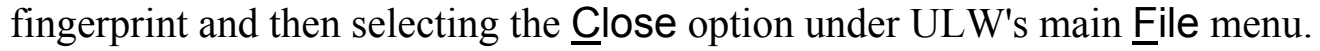


A new session is then opened into which the image on the Clipboard will be manually pasted. To start a new ULW transaction session, select the New Latent Feature Search (LFFS) option under ULW's main File menu as shown in Figure 11.

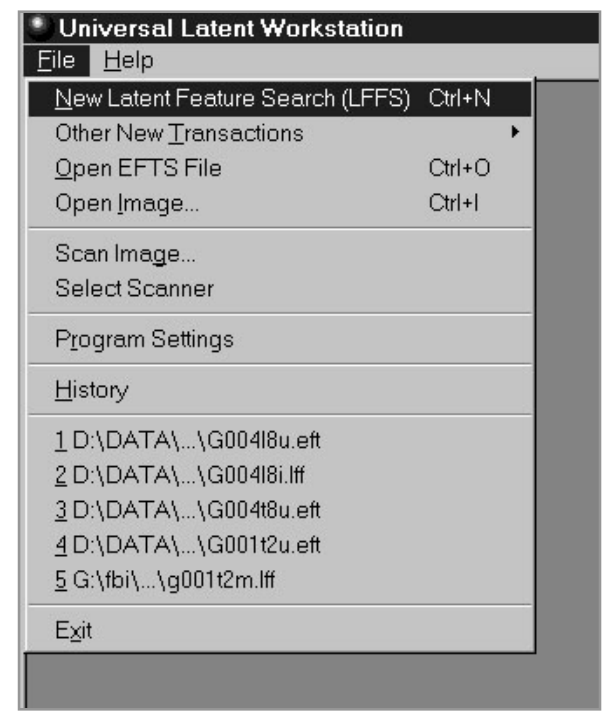

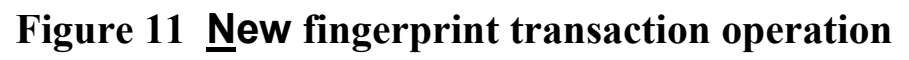

\subsection{Paste Clipboard Image into ULW}

As can be seen in the left column below, ULW creates a new transaction session, including a default Type-1 record along with an empty Type- 2 descriptive record, and an empty Type- 9 minutiae record. The Subject Description fields of the Type-2 record are automatically listed to the right.

To import the image on the Clipboard, first click on the FBI IAFIS option under the LFFS list on the left as shown.

\begin{tabular}{|c|c|c|c|c|c|}
\hline \multicolumn{6}{|c|}{ Universal Latent Workstation - [LFFS: (No image)] } \\
\hline LFFS: (No image) & Name & Abbr & Field & $\#$ & Value \\
\hline \multirow{4}{*}{ 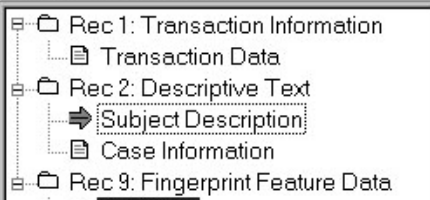 } & Gender & SEX & 2.024 & & \\
\hline & Race & RAC & 2.025 & & \\
\hline & Age Range & AGR & 2.023 & & \\
\hline & Height Range & HTR & 2.028 & & \\
\hline \multirow{2}{*}{$\begin{array}{l}\text { - FBI IAFIS } \\
\text { ○ Cogent (empty) }\end{array}$} & Weight Range & WTR & 2.030 & & \\
\hline & Hair Color & $\mathrm{HAl}$ & 2.032 & & \\
\hline \multirow{4}{*}{$\begin{array}{l}\text { O Morpho (empty) } \\
\text { O NEC (empty) } \\
\text { o Printrak (empty) } \\
\text { 国 Annotations }\end{array}$} & Color Eyes & EYE & 2.031 & & \\
\hline & Scars Marks \& Tattoos & SMT & 2.026 & 1 & \\
\hline & Place of Birth & $\mathrm{POB}$ & 2.020 & & \\
\hline & Geographical Area of Search & GEO & 2.044 & 1 & \\
\hline
\end{tabular}

Figure 12. New fingerprint transaction session 
Upon clicking FBI IAFIS, ULW will attempt to display a fingerprint image in the right portion of its screen and overlay any minutiae recorded in the Type-9 record. Because there is no image record currently associated with the transaction, a text window reading "(No image loaded) " pops up in the right window was shown in Figure 13.

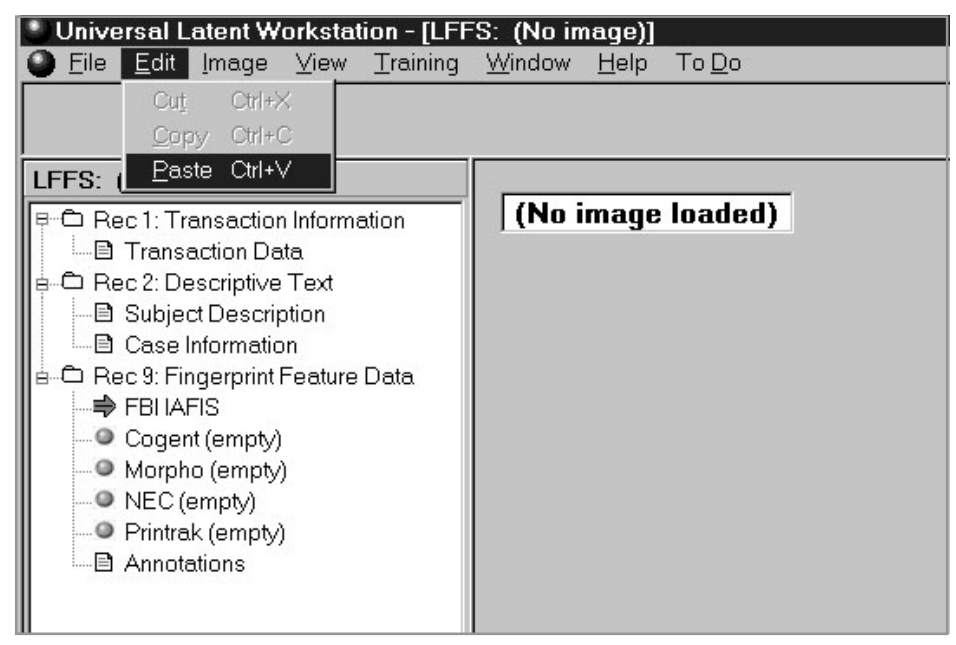

Figure 13. Empty fingerprint transaction

To paste the fingerprint image on the Clipboard into this transaction session, select the Paste option under the main Edit menu as shown above. The image is displayed in the right portion of the ULW screen, and immediately an Image Resolution widow (like the one shown below) pops up asking for the image's scan resolution to be specified. For SD7 images, click the last button at the bottom of the window, signifying the image was scanned at 19.69 pixels per millimeter (500 pixels per inch).

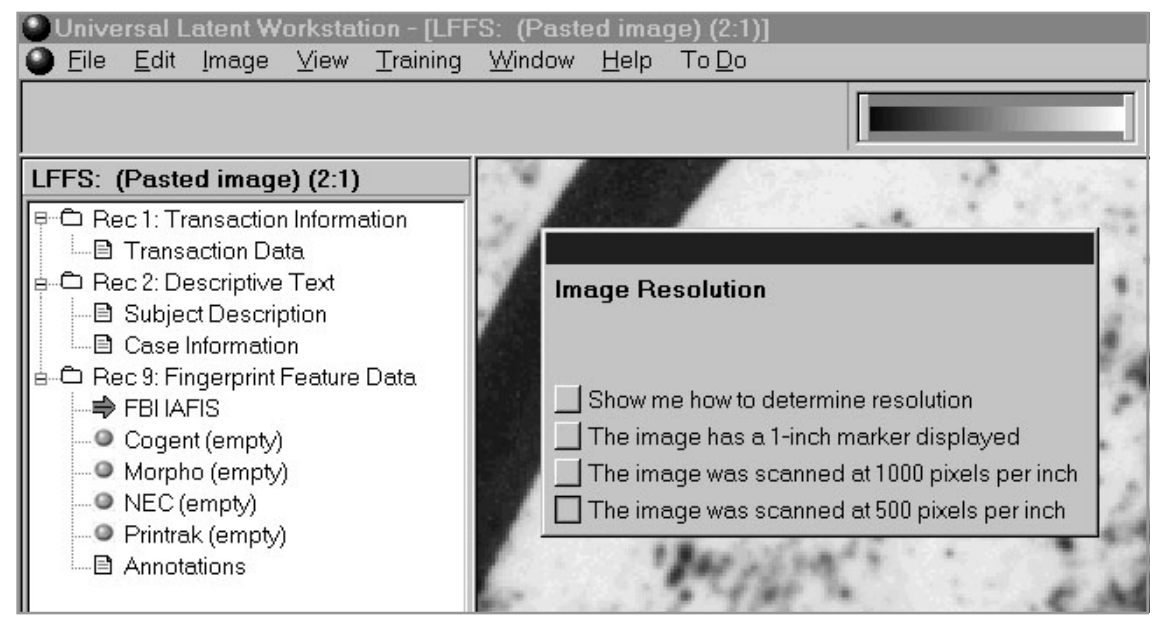

Figure 14. Image Resolution window 
Upon selecting the resolution, the pop-up window disappears, and ULW now has inserted a new Type- 4 record, containing the pasted image, into the transaction. The results are shown in Figure 15.

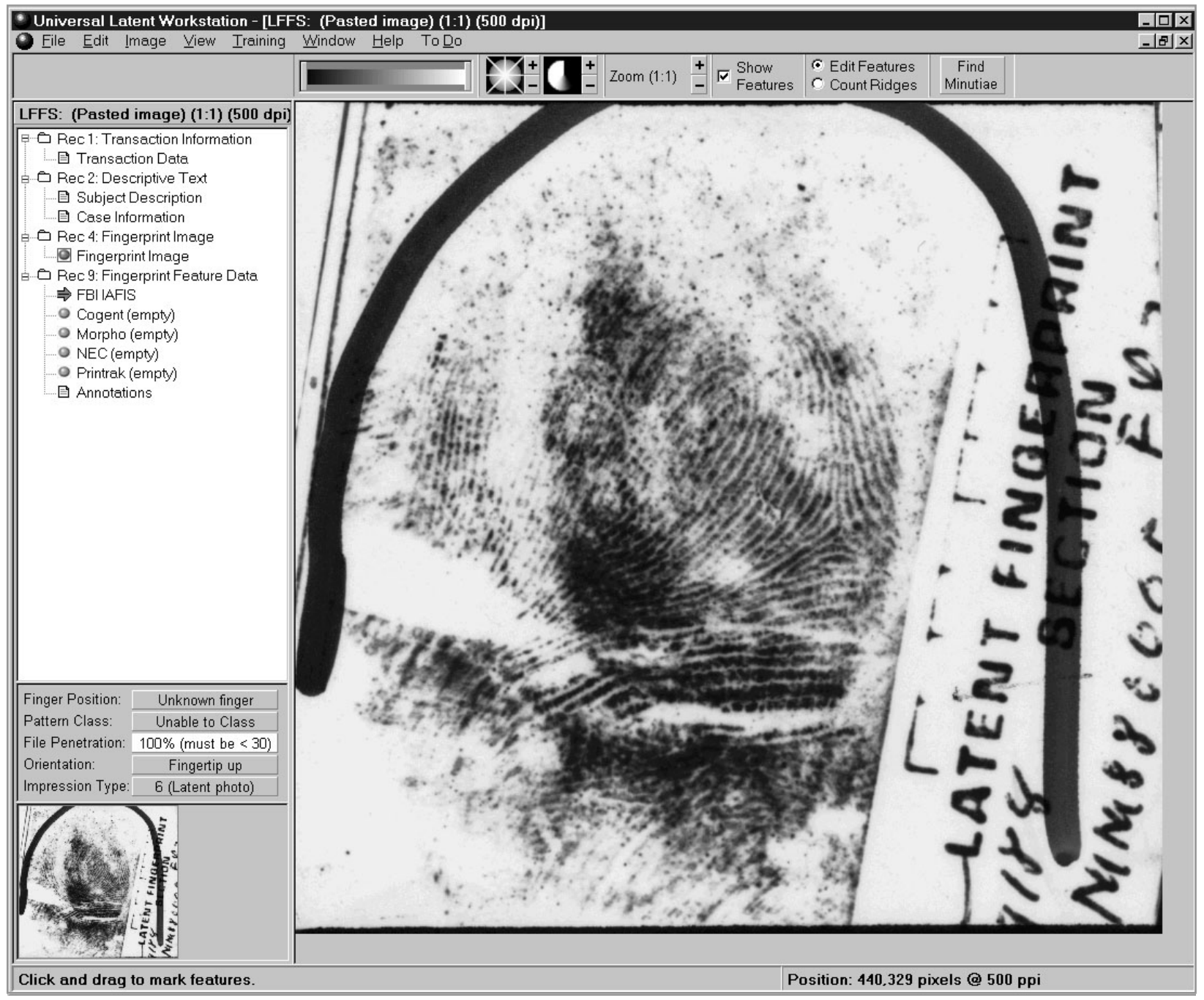

Figure 15. Clipboard image pasted into ULW transaction

\subsection{Save LFFS Transaction [Optional]}

ULW is now prepared to be used to mark (encode) the minutiae on the fingerprint image. (See ULW documentation for details.) Due to the number of steps taken to get to this point, it is a good idea to save the transaction to a new ANSI/NIST file. Although, this step is optional.

From the main File menu in ULW, select the Save As option as shown Figure 16. ULW checks to make sure all mandatory Type- 2 descriptive fields have been assigned values. If prompted by a pop-up window that says, "There are errors in the submission. Do you wish to save anyway?," either the fields that have been flagged in ULW should be filled in or simply click Yes and they will be ignored. If minutiae have been added to the image, ULW checks to make sure their intervening ridge counts have been verified. If prompted by a 
pop-up window that says, "Please note that ridge counts have not been manually verified ... Do you wish to continue?," either stop and verify the ridge counts or click $\underline{Y}$ es and this too will be ignored.

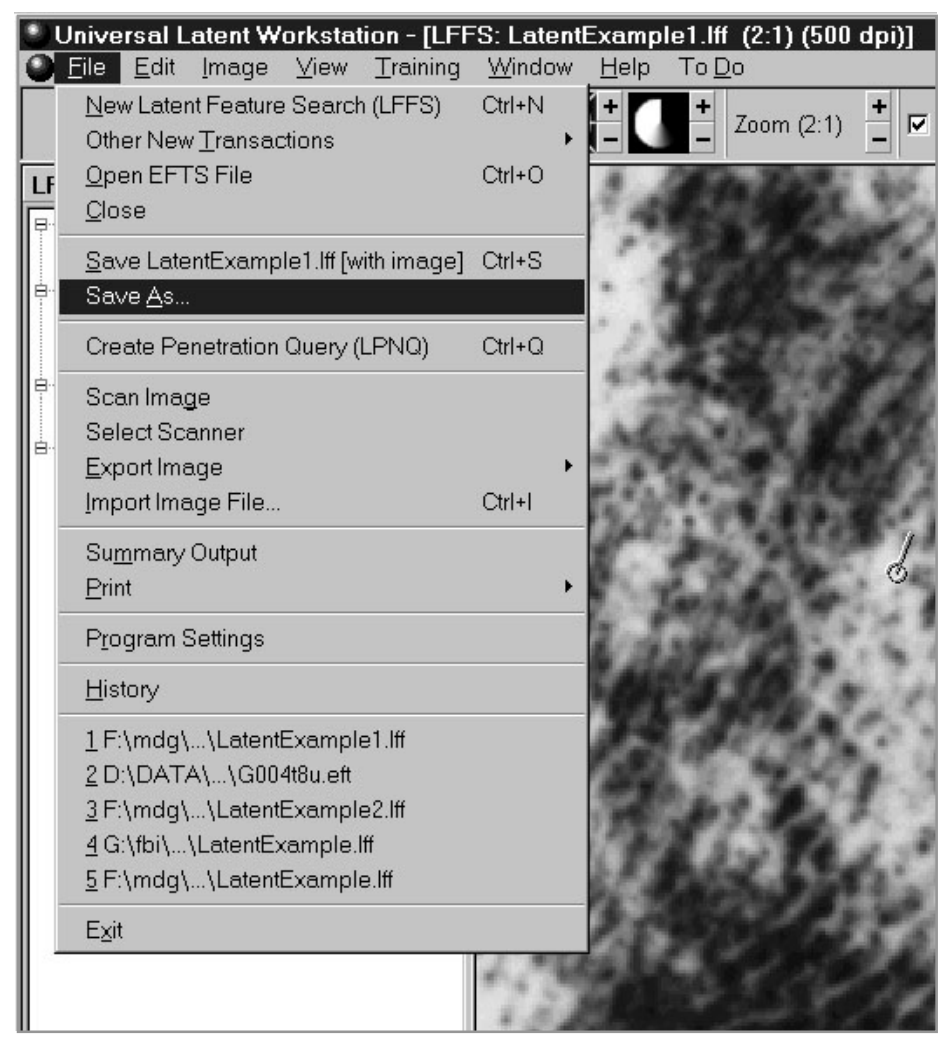

Figure 16. Save As operation

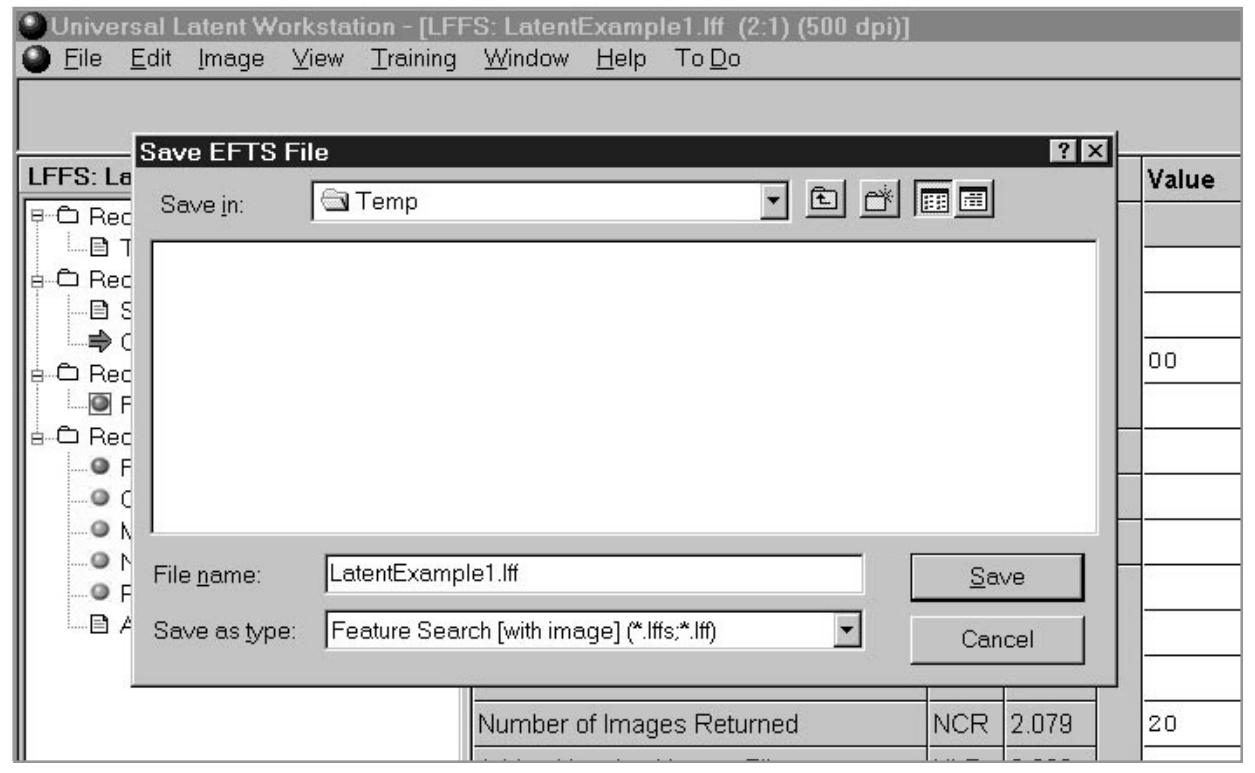

Figure 17 Saving a fingerprint transaction to file 
A Windows Explorer dialogue box will pop up like the one shown in Figure 17. Designate a destination folder similar to the Open EFTS File operation earlier. In this example, a temporary folder Temp has been chosen. Enter the name of the file to be created or overwritten, in this case LatentExample1. Iff. Then, click the Save button. The current transaction is now saved to the designated file and can be loaded back into ULW later using the Oppen EFTS File operation.

\section{USING SD27 TRAINING DATA}

At this point, ULW may be used just as if the image were scanned into the system. The operator can enhance the image on the screen, select an area of interest, run automatic minutiae detection, and manually add or remove cores, deltas, and minutiae. If a complete transaction is desired, then ridge counts should be verified, descriptive data will need to be entered for the Type- 2 record(s), and search criteria will need to be specified to bring the File Penetration level below $30 \%$. (These topics, while important, are not relevant to the purpose of this report.) Figure 18 shows an example of a marked fingerprint in ULW. Note the image has been zoomed up 2 to 1.

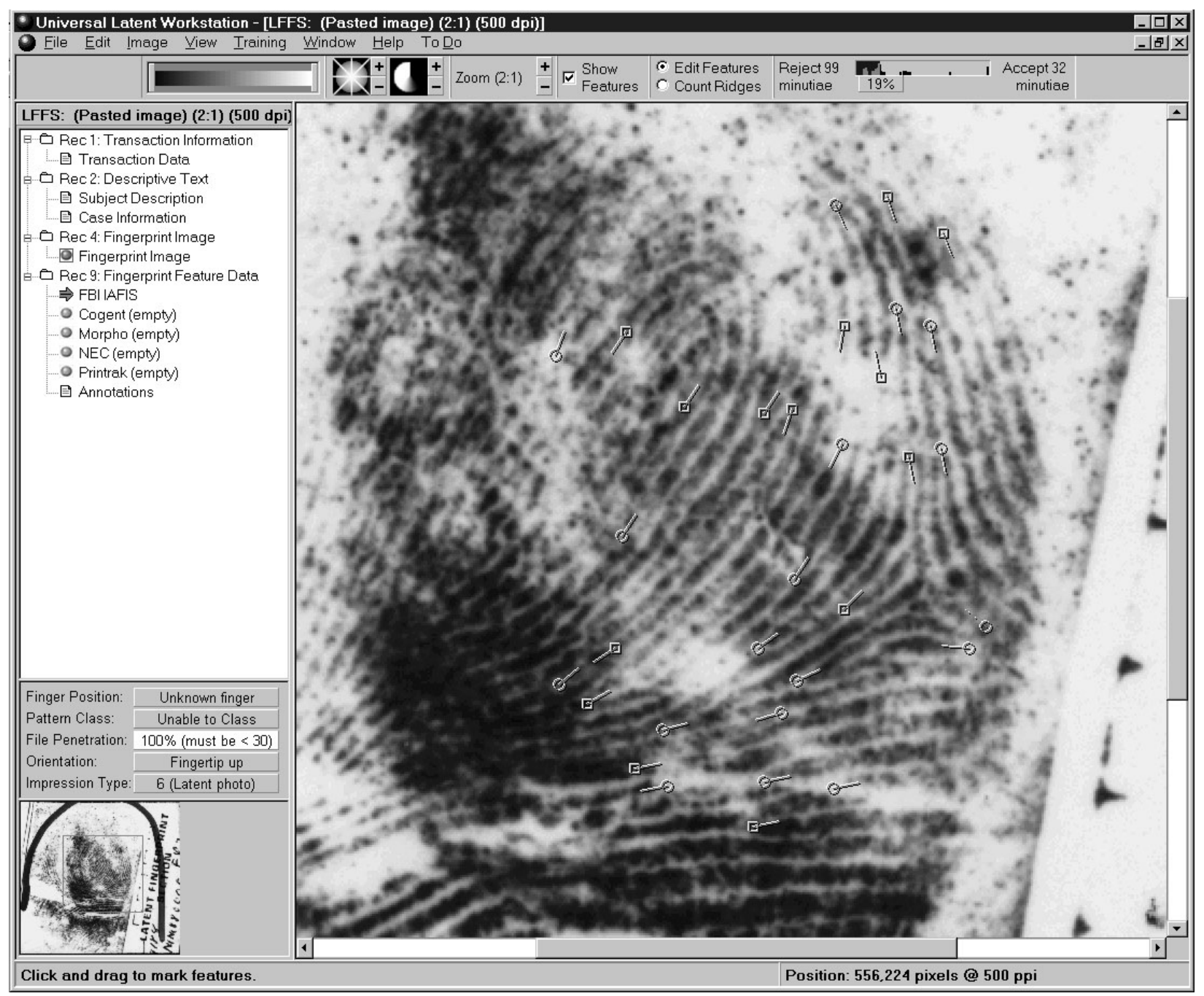

Figure 18. Example of encoded latent fingerprint 


\subsection{Overlay Desired SD27 Minutiae}

All ANSI/NIST files in SD27 with filename extension "lff" contain Type-9 minutiae records. There are four sets of minutiae in each case folder in the distribution. Of these, there are two ideal sets, one corresponding to all visible minutiae on the latent image, and the other corresponding to all visible minutiae on the tenprint mate's image. There are also two matched sets, each containing only the minutiae that are in common between the latent and the tenprint images. As mentioned earlier, the ideal sets will likely be more useful for training fingerprint examiners.

Once an SD27 fingerprint image is loaded into an ULW transaction, and an attempt has been made to label all the minutiae in the image, professionally validated minutiae can be overlaid for instantaneous visual comparison. This serves as a powerful training tool.

To overlay a set of SD27 minutiae, select the Read File to Compare option under the main Training menu in ULW as shown Figure 19.

Now choose the SD27 file with the training minutiae of interest. In this example, the latent image from case G004 has been encoded. Therefore, the case folder G004 on the SD27 CDROM has been opened, and the file G004l8i. Iff is selected in Figure 20. This ANSI/NIST file contains the ideal set of minutiae for the latent image. (Please refer to the file naming convention in Figure 4.)

Click the Open button to overlay the selected set of minutiae. ULW plots the comparison minutiae on top the labeled fingerprint image and then immediately displays a Feature Set Comparison window as shown in Figure 21. In this window, ULW compares the trainee's minutiae against the overlaid set and posts the number of matched, missed, and false minutiae, and it includes a graphic symbolically depicting the distance between matched pairs of minutiae. (For more information on the Feature Set Comparison window, select the Help option at the top of the window.) To close this window, click the close button (marked X) at the top right of the window.

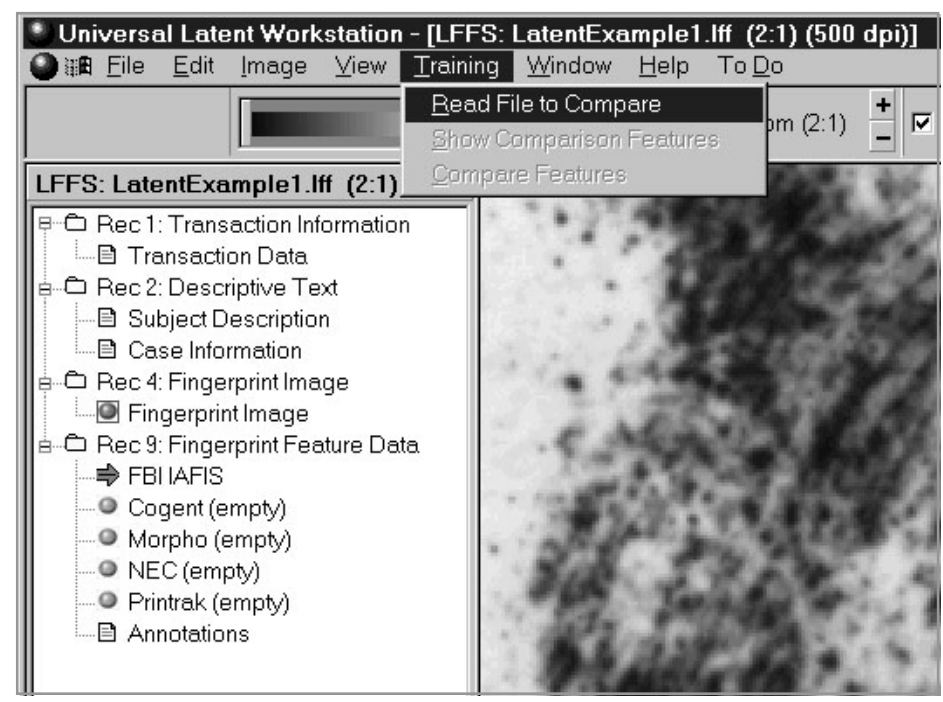

Figure 19. Read training data operation 


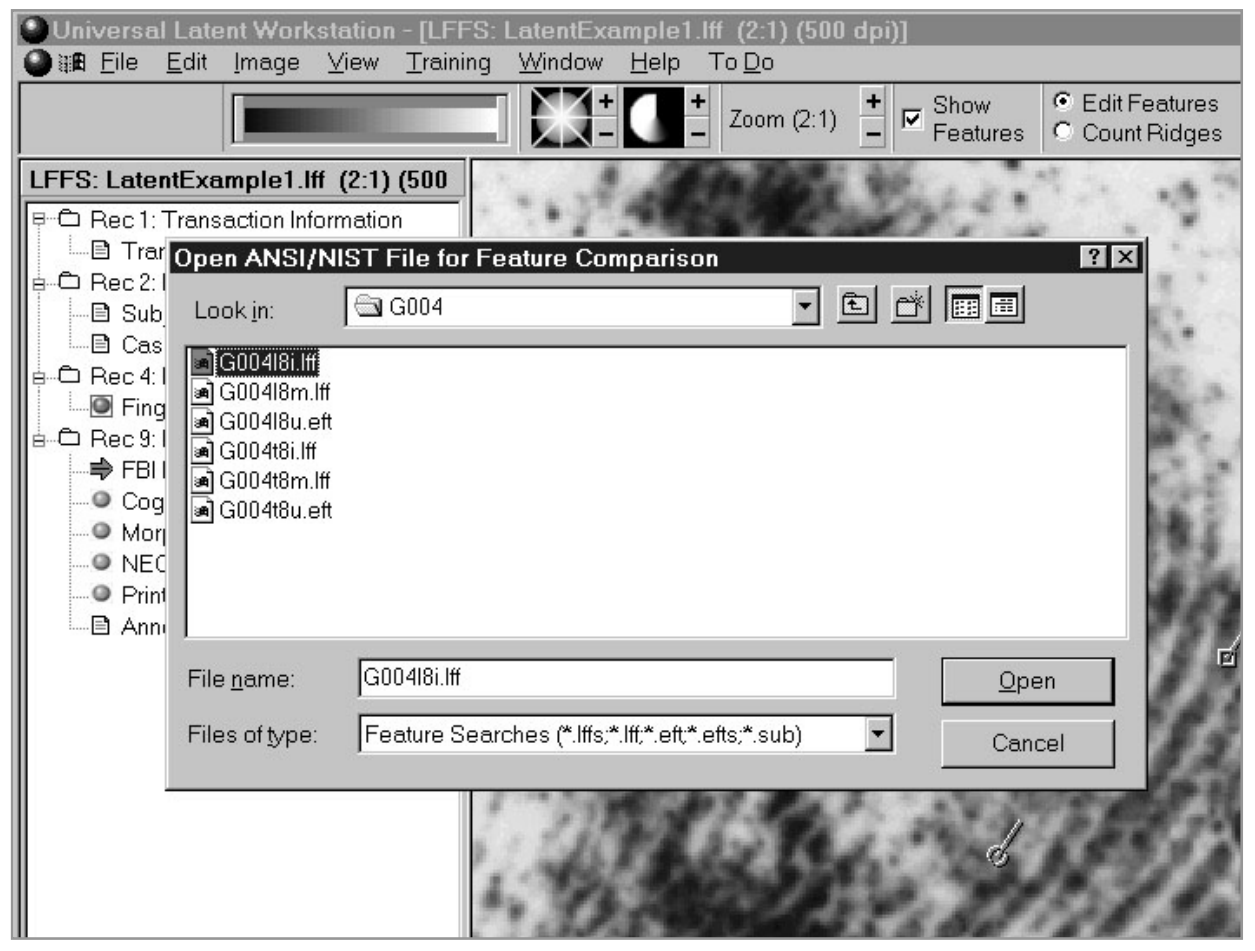

Figure 20. Select minutiae set for overlay

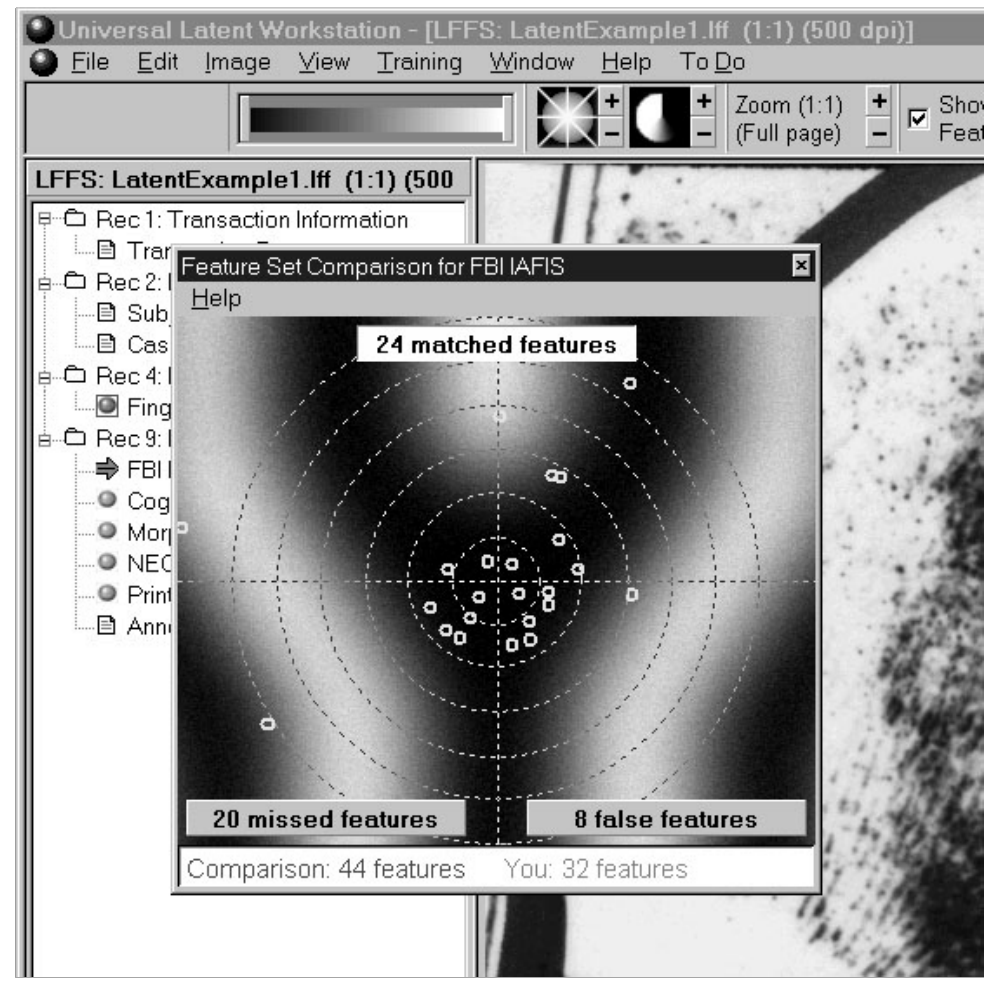

Figure 21. Feature Set Comparison window 
The labeled fingerprint in Figure 18, is overlaid with the selected ideal set of minutiae and shown below. Unfortunately, it is very difficult to distinguish the trainee's features in this grayscale figure from the SD27 training features. In ULW, the distinction is apparent as the default color of the trainee's features is red and the default color of the SD27 training features is blue.

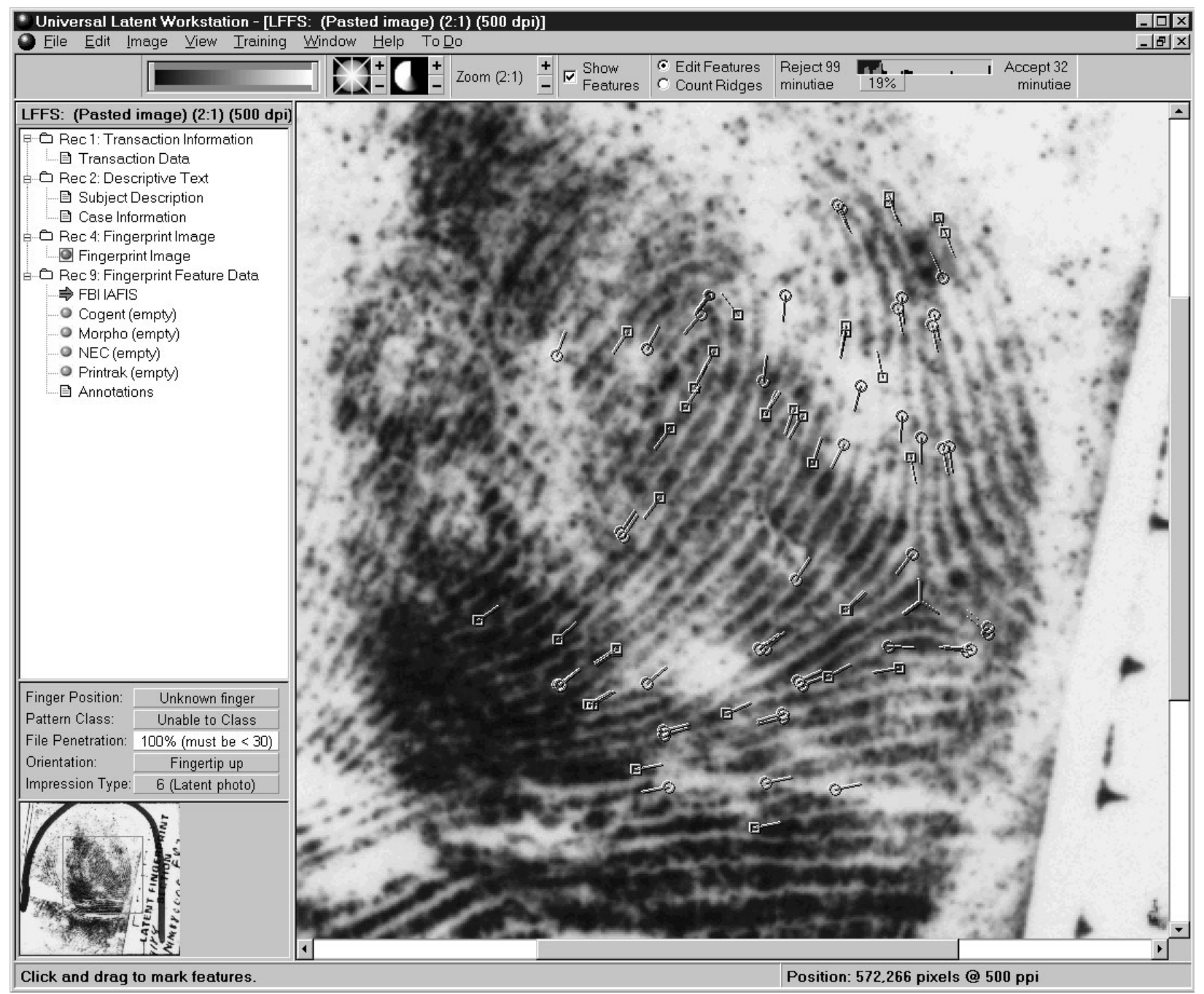

Figure 22. Encoded fingerprint with SD27 training minutiae overlaid

The distinction between sets of minutiae can be made clearer by bringing up two separate images of the same fingerprint simultaneously in ULW. The results are shown in Figure 23. To do this, the latent fingerprint transaction had to be stored to two separate files, in this case LatentExample1.lff and LatentExample2.lff, and then both were loaded individually using the Oppen EFTS File operation described earlier. The minutiae on the left image were manually labeled by the trainee, while the minutiae on the right image were overlaid using the Read File to Compare option under the main Iraining menu in ULW.

To display multiple images simultaneously side by side in ULW, load multiple transaction files,

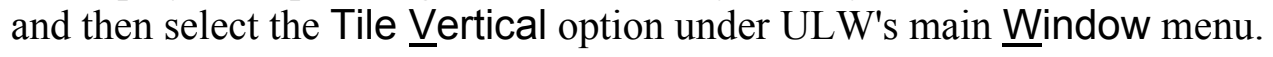




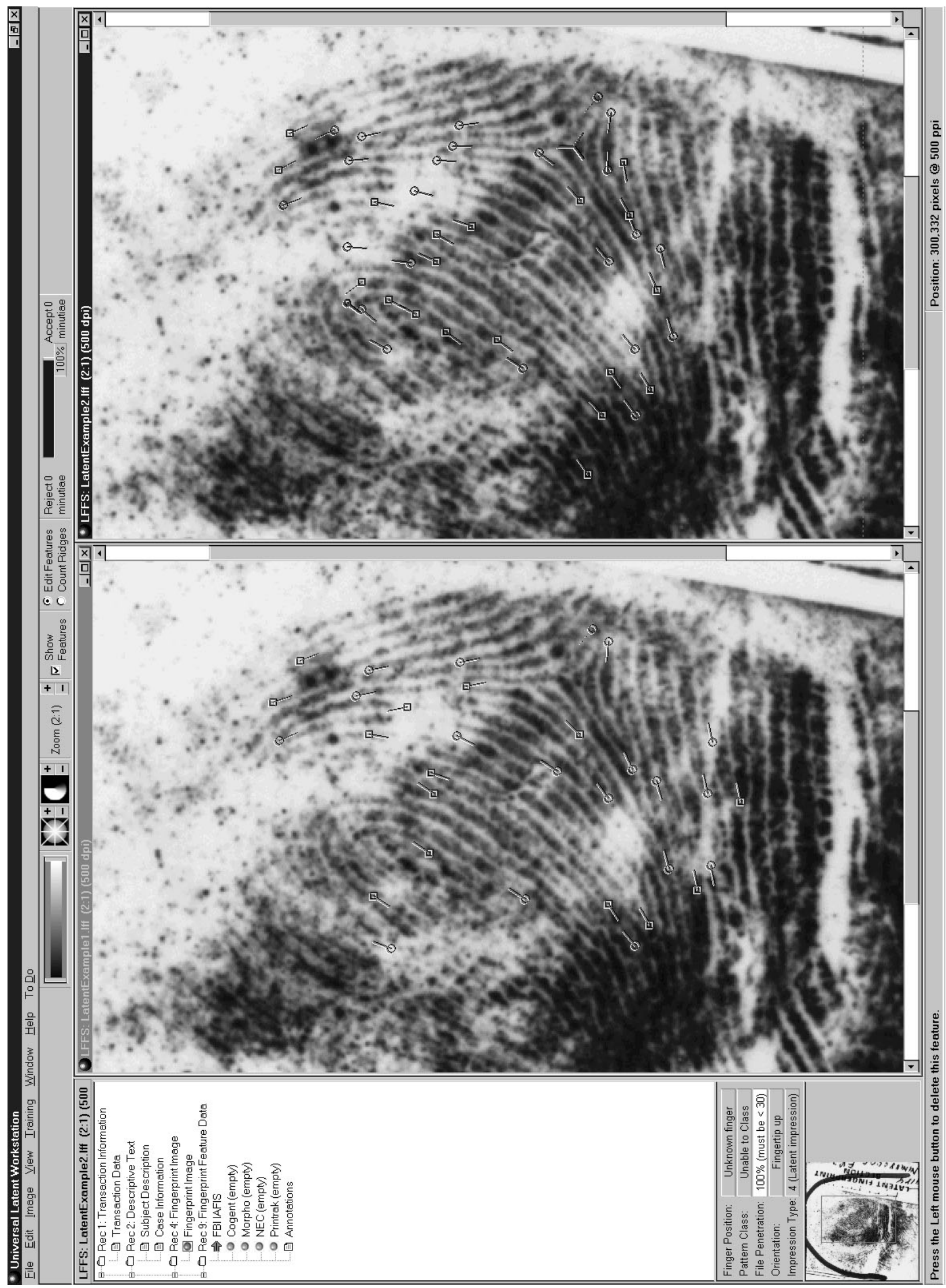

Figure 23. Left - trainee results Right - SD27 training minutiae 


\subsection{Latent to Tenprint Comparison}

It is also possible to load the latent image and its tenprint mate simultaneously in ULW. To do this, the steps in Section 2 must be followed for each image. Figure 24 shows the results of loading both the latent and tenprint mate fingerprints for SD27 case G004 into ULW. Each image has its corresponding set of matched minutiae overlaid as training data. (Notice that no distinction is made between ridge endings and bifurcations in the matched sets of minutiae.) 


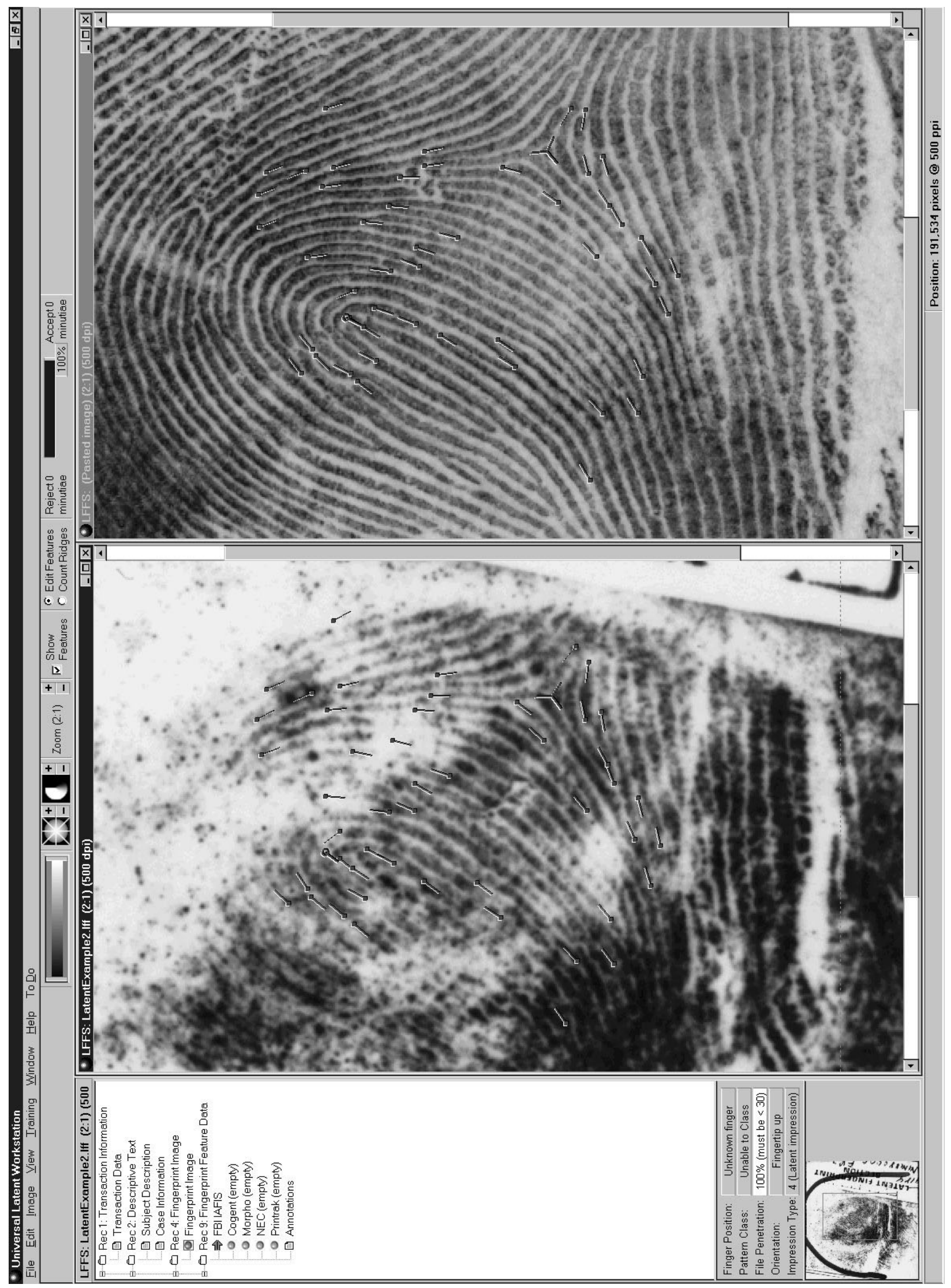

Figure 24. Matched minutiae on latent (left) and its tenprint mate (right) 


\section{SUMMARY}

A simple training scenario has been presented using SD27 in conjunction with ULW. Steps for loading an SD27 fingerprint image into ULW and for overlaying SD27 validated minutiae were described. Once the fundamental steps presented in this report are mastered, different combinations of displayed images with different combinations of trainee-provided and SD27overlaid minutiae sets are possible. Used together, these resources can pose an effective and low-cost method for training fingerprint examiners.

\section{REFERENCES}

[1] M.D. Garris \& R.M. McCabe, "NIST Special Database 27: Fingerprint Minutiae from Latent and Matching Tenprint Images," NIST Technical Report NISTIR 6534 \& CD-ROM, June 2000.

[2] J.H. Wegstein, “A Semi-automated Single Fingerprint Identification System”, NBS Technical Note 481, April 1969.

[3] J.H. Wegstein, “Automated Fingerprint Identification”, NBS Technical Note 538, August 1970 .

[4] R.T. Moore, "The Influence of Ink on The Quality of Fingerprint Impressions", NBS Technical Report NBSIR 74-627, December 1974.

[5] J.H. Wegstein, “The M40 Fingerprint Matcher”, NBS Technical Note 878, July 1975.

[6] J.H. Wegstein, and J.F. Rafferty, “The LX39 latent Fingerprint Matcher”, NBS Special Publication 500-36, August 1978.

[7] R.T. Moore, "Results of Fingerprint Image Quality Experiments", NBS Technical Report NBSIR 81-2298, June 1981.

[8] J.H. Wegstein, “An Automated Fingerprint Identification System”, NBS Special Publication 500-89, February 1982.

[9] R.T. Moore, "Automated Fingerprint Identification Systems - Benchmark Test of Relative Performance”, American National Standard ANSI/IAI 1-1988, February 1988.

[10] R.M. McCabe, "Data Format for the Interchange of Fingerprint, Facial, Scar Mark \& Tattoo (SMT) Information," American National Standard ANSI/NIST-ITL 1-2000, July 2000. Available from R.M. McCabe at NIST, 100 Bureau Drive, Stop 8940, Gaithersburg, MD 208998940.

[11] R.M. McCabe, and R.T. Moore, "Data Format for Information Interchange", American National Standard ANSI/NBS-ICST 1-1986, August 1986.

[12] R.M. McCabe, "Data Format for the Interchange of Fingerprint Information", American National Standard ANSI/NIST-CSL 1-1993, November 1993.

[13] "Electronic Fingerprint Transmission Specification," CJIS-RS-0010 (V7). Available from Criminal Justice Information Services Division, Federal Bureau of Investigation, 935 Pennsylvania Avenue, NW, Washington D.C. 20535. 